\title{
Critical Metal Phase at the Anderson Metal-Insulator Transition with Kondo Impurities
}

\author{
S. Kettemann, ${ }^{1}$ E. R. Mucciolo, ${ }^{2}$ and I. Varga ${ }^{3}$ \\ ${ }^{1}$ Jacobs University, School of Engineering and Science, Campus Ring 1, 28759 Bremen, \\ Germany, and Division of Advanced Materials Science Pohang University of Science and \\ Technology (POSTECH) San31, Hyoja-dong, Nam-gu, Pohang 790-784, South Korea \\ ${ }^{2}$ Department of Physics, University of Central Florida, P. O. Box 162385, Orlando, FL 32816, USA \\ ${ }^{3}$ Elméleti Fizika Tanszék, Budapesti Müszaki és Gazdaságtudományi Egyetem, H-1521 Budapest, Hungary
}

(Dated: February 28, 2022)

\begin{abstract}
It is well-known that magnetic impurities can change the symmetry class of disordered metallic systems by breaking spin and time-reversal symmetry. At low temperature these symmetries can be restored by Kondo screening. It is also known that at the Anderson metal-insulator transition, wave functions develop multifractal fluctuations with power law correlations. Here, we consider the interplay of these two effects. We show that multifractal correlations open local pseudogaps at the Fermi energy at some random positions in space. When dilute magnetic impurities are at these locations, Kondo screening is strongly suppressed. We find that when the exchange coupling $J$ is smaller than a certain value $J^{*}$, the metal-insulator transition point extends to a critical region in the disorder strength parameter and to a band of critical states. The width of this critical region increases with a power of the concentration of magnetic impurities.
\end{abstract}

Fifty years after its proposal, the Anderson metal-insulator transition (AMIT) of disordered noninteracting electrons [1] continues to be intensively studied [2, 3, 4, 5]. The AMIT is a quantum phase transition of second order where the localization length diverges with a critical exponent $\nu$. The critical state is multifractal and characterized by a wide distribution of wave function amplitudes with a log-normal shape [5]. One aspect which remains less understood is the interplay between the AMIT of conduction electrons and the dynamics of the spin of magnetic impurities. These can be induced by energy levels below the Fermi energy such as the d-levels of transition metal impurities [6] or by localized electronic states such as the dopant levels in semiconductors [7]. Magnetic moments can enhance the spin susceptibility and the specific heat as has been observed in Si:P close to the AMIT [8, 9]. When local magnetic moments interact with the conduction electrons by an exchange interaction, they can break both spin degeneracy and time-reversal symmetry (TRS) of the conduction electrons. Therefore, they are expected to change the symmetry class of the electronic system from orthogonal to unitary [10], changing the critical exponent $\nu$, the critical electron density $n_{c}$, and the critical disorder strength $W_{c}$ at which the transition occurs [11, 12]. At sufficiently low temperatures, an additional effect comes into play. The antiferromagnetic exchange interaction between spin- $1 / 2$ local moments and the conduction electrons in a metal leads to a correlated electron state where a Kondo singlet is formed, screening the local moments at temperatures below the Kondo temperature $T_{K}$. In this limit, the magnetic susceptibility $\chi$ approaches a constant value, as indicated in the left inset of Fig. 11 and the magnetic moments cease to break the TRS of the conduction electrons. The situation is quite different in the insulating phase, where local spectral gaps $\Delta_{I}$ prevent the development of the Kondo screening whenever the exchange coupling $J$ between local moments and conduction electrons is below a critical value $J_{c}^{A}$ [13]. In this case, the unscreened, free magnetic moments (FMMs) yield a low-temperature susceptibility $\chi \sim 1 / T$, as

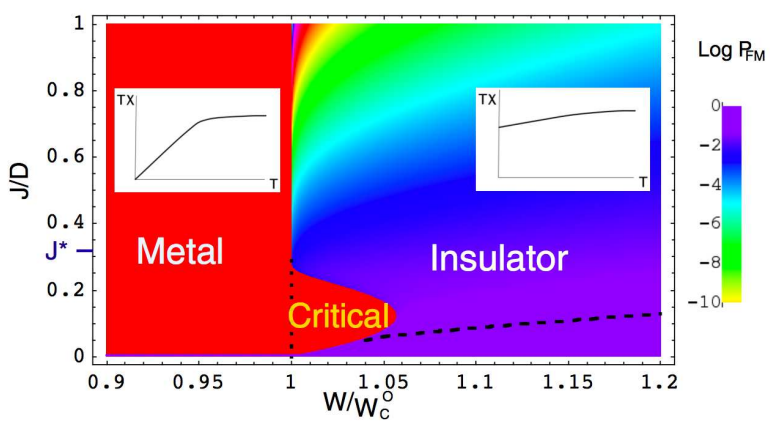

FIG. 1: (Color online) The fraction of free magnetic moments $P_{\mathrm{FM}}$ [Eq. [13] in a three-dimensional disordered electron system as function of the exchange coupling $J$ (in units of band width $D$ ) and the disorder strength $W$ (in units of the critical value $W_{\mathrm{c}}^{\mathrm{O}}$ ). Dashed line: $J_{c}^{A}(W)$, Eq. [5, the critical exchange coupling caused by local spectral gaps due to Anderson localization. For $J<J^{*} \approx 0.3 D$, there is a critical metal region for $W_{\mathrm{c}}^{\mathrm{O}}<W<W_{\mathrm{c}}(J)$, Eq. 14. Insets: temperature dependence of the local magnetic susceptibility $\chi$ of a single magnetic impurity in the metallic and in the insulating phase, showing Kondo screening and free moment behavior, respectively.

indicated in the right inset of Fig. 11 As a result, TRS may be partially broken in the insulating phase. The symmetry class at the AMIT is determined by the subtle interplay between the Kondo screening and the Anderson localization.

In this Letter, we aim at determining the density of FMMs in the vicinity of the AMIT in the low-temperature limit $T \ll\left\langle T_{K}\right\rangle$ for a system of dilute magnetic impurities with an average Kondo temperature $\left\langle T_{K}\right\rangle$. An essential aspect of the problem are the multifractal fluctuations of the eigenfunctions at the AMIT. They lead to the local suppression of wave function amplitudes at random locations [14]. Multifractal states are power-law correlated over a large energy interval $E_{c}$ of the order of the band width $D[15,16]$. We show that these spectral correlations induce local pseudogaps which, in turn, prevent the Kondo screening of a sizeable fraction of local 
moments even when the exchange coupling exceeds $J_{c}^{A}$ (see Fig. 1). When the exchange coupling $J$ is sufficiently weak, these FMMs can break TRS and shift the transition to stronger disorder amplitudes, $W_{c}(J)$, as well as lower the critical exponents on the insulating side to their unitary values. Coming from the metallic side, the transition occurs at the smaller disorder strength $W_{c}^{O}$ since the magnetic impurities become Kondo screened. We conclude that the critical point is extended to a critical region, as shown in Fig. 1.

The most general model for the formation of magnetic impurities is the Anderson model [6] describing a localized level with energy $\epsilon_{d}$ and on-site Coulomb repulsion $U$ hybridizing with electrons in the conduction band of a Hamiltonian $H_{0}$ which may include the random potential caused by nonmagnetic impurities $V(\mathbf{r})$. Using as basis the eigenstates $\psi_{n}$ with energy $E_{n}$ of $H_{0}$ and the density operator $\hat{n}_{n, \sigma}$, the Anderson Hamiltonian can be written as

$$
\begin{aligned}
H_{A}= & \sum_{n, \sigma} E_{n} \hat{n}_{n, \sigma}+\epsilon_{d} \sum_{\sigma} \hat{n}_{d, \sigma}+U \hat{n}_{d,+} \hat{n}_{d,-} \\
& +\sum_{n, \sigma}\left(t_{n d} c_{n \sigma}^{+} d_{\sigma}+\text { H.c. }\right) .
\end{aligned}
$$

Here, $\hat{n}_{d, \sigma}$ is the density operator in the impurity level. The hybridization amplitude $t_{n d}$ is proportional to the eigenfunction amplitude at the position of the magnetic impurity $\psi_{n}^{*}(0)$ and the localized orbital amplitude $\phi_{d}(0): t_{n d}=$ $t \psi_{n}^{*}(0) \phi_{d}(0)$. One can employ a Schrieffer-Wolff transformation [17, 18] formulated in terms of eigenstates $\psi_{n}$ [19] to generate an s-d Hamiltonian with exchange couplings

$$
J_{n n^{\prime}}=t_{n d}^{*} t_{n^{\prime} d}\left(\frac{1}{U+\epsilon_{d}-E_{n^{\prime}}}+\frac{1}{E_{n}-\epsilon_{d}}\right)
$$

and an additional potential scattering term with amplitude

$$
K_{n n^{\prime}}=t_{n d}^{*} t_{n^{\prime} d}\left(-\frac{1}{U+\epsilon_{d}-E_{n^{\prime}}}+\frac{1}{E_{n}-\epsilon_{d}}\right) .
$$

Note that $K_{n n^{\prime}}$ vanishes for all $n, n^{\prime}$ when $\left|E_{n, n^{\prime}}-E_{F}\right| \ll U$ and $\epsilon_{d}=E_{F}-U / 2$, where $E_{F}$ is the Fermi energy. For arbitrary $\epsilon_{d}$ wave functions with small amplitude at the position of the magnetic impurity are hardly modified by this potential scattering term since $K_{n n^{\prime}} \sim \psi_{n}^{*}(0) \psi_{n^{\prime}}(0)$ and we can disregard it when deriving the density of FMMs. Thus, we retain only the exchange couplings $J_{n n^{\prime}}=J \psi_{n}^{*}(0) \psi_{n^{\prime}}(0)$.

Critical exchange coupling. - Recently, it was shown that the distribution of the exchange couplings $J_{n n}$ results in a wide bimodal distribution of the Kondo temperature and free magnetic moments [9, 19, 20, 21]. This was at first obtained by solving the 1-loop Nagaoka-Suhl equation (NSE) [22] of the Kondo model in the representation of the eigenstates of $H_{0}$. It was confirmed with the numerical renormalization group and the continuous time quantum Monte Carlo method [21]. These nonperturbative methods show that the NSE underestimates the density of FMMs. Building on that work, we can get the lower limit of the critical exchange coupling $J_{c}$ by setting $T_{K} \rightarrow 0$ in the NSE:

$$
\frac{1}{J_{c}}=\frac{1}{2 N} \sum_{n=1}^{N} \frac{L^{d}\left|\psi_{n}(0)\right|^{2}}{\left|E_{n}-E_{F}\right|}
$$

where $L$ denotes the linear size of the system, $N \sim L^{d}$ the number of states in the band, and $d$ is the spatial dimension. For a clean metallic system with a flat band, the critical exchange coupling vanishes logarithmically with the number of states $N$ as $J_{c} \sim D / \ln N$, leaving no FMMs for any finite value of $J$. On the other hand, if the eigenstates at the Fermi energy become localized with a localization length $\xi$, finite local gaps of order $\Delta_{I}=\left(\rho \xi^{d}\right)^{-1}$ appear at the Fermi energy, cutting off the Kondo renormalization [13] ( $\rho$ is the average density of states). Thus, there are FMMs whenever $\Delta_{I} \gg T_{K}$, or, equivalently, $J \ll J_{c}^{\mathrm{A}} \sim D / \ln N_{I}$, where $N_{\mathrm{I}}=D / \Delta_{\mathrm{I}}$ is the number of localized states with a finite wave function amplitude at the magnetic impurity site [21].

Critical exchange coupling at the AMIT. - For $d>2$ an AMIT exists and the localization length diverges at the mobility edge $E_{\mathrm{ME}}$ as a power law, $\xi(E) \sim\left(\left|E-E_{\mathrm{ME}}\right| / E_{c}\right)^{-\nu}$. Another way to probe the transition region is to vary the disorder strength $W$. At a critical strength $W_{c}$, the localization length diverges with the same exponent $\nu$. Thus, one can draw a quantum phase diagram with $J$ versus $W$ where the FMM phase is limited by a line defined by the function

$$
J_{c}^{A}(W) / D=\left[\nu d \ln \left(E_{c} /\left|W-W_{c}\right|\right)\right]^{-1},
$$

as shown by the dashed line in Fig. 1.

Local Pseudo Gaps. - Equation (5) does not take into account the multifractality and the critical correlations between wave functions at different energies at the AMIT [15]. The amplitude of multifractal states is suppressed at some random positions below their typical value, scaling as $L^{-\alpha}$ with $\alpha>d$ (i.e., decaying faster than extended states). Correlations between wave functions at different energies can then open wide local pseudogaps. These correlations can be quantified by spatially integrating the correlation function of eigenfunction probabilities associated with two energy levels distant in energy by $\omega_{n m}=E_{n}-E_{m}$, namely [16],

$$
\begin{aligned}
C_{n m} & =L^{d} \int d^{d} r\left\langle\left|\psi_{n}(\mathbf{r})\right|^{2}\left|\psi_{m}(\mathbf{r})\right|^{2}\right\rangle \\
& = \begin{cases}\left(E_{c} / \Delta\right)^{\gamma}, & \left|\omega_{n m}\right|<\Delta, \\
\left(E_{c} /\left|\omega_{n m}\right|\right)^{\gamma}, & \Delta<\left|\omega_{n m}\right|<E_{c}, \\
\left(E_{c} /\left|\omega_{n m}\right|\right)^{2}, & \left|\omega_{n m}\right|>E_{c},\end{cases}
\end{aligned}
$$

where $0<\gamma<1$. For $\omega_{n m}<E_{c}$ the value of $C_{n m}$ is enhanced in comparison to the plane-wave limit $C_{n m}=1$. The distribution function of a multifractal wave function is radically different from the Porter-Thomas distribution of metallic states since the moments of eigenfunction intensities $\left|\psi_{l}(\mathbf{r})\right|^{2}$ obey the power-law scaling $P_{q}=L^{d}\left\langle\left|\psi_{l}(\mathbf{r})\right|^{2 q}\right\rangle \sim L^{-\tau_{q}}$, where $\tau_{q}$ is the multifractal exponent of the $q$-th moment. The corresponding distribution function is known to be lognormal, in good approximation [5],

$$
P(\alpha)=L^{-\frac{\left(\alpha-\alpha_{0}\right)^{2}}{2 d \gamma}},
$$


where $\alpha=-\ln \left|\psi_{l}(\mathbf{r})\right|^{2} / \ln L . \alpha_{0}>d$ is related to the multifractal exponents, $\tau_{q}=d(q-1)+\left(\alpha_{0}-d\right) q(1-q)$, and to $\gamma=2\left(\alpha_{0}-d\right) / d$. In order to find $J_{c}$ we need the joint distribution of two eigenfunction intensities $\left|\psi_{l}(\mathbf{r})\right|^{2}$ and $\left|\psi_{n}(\mathbf{r})\right|^{2}$, $l \neq n$ which is also log-normal,

$$
P\left(\alpha_{l}, \alpha_{k}\right)=L^{-2 d+a_{l k}\left[f\left(\alpha_{l}\right)+f\left(\alpha_{k}\right)\right]+b_{l k} \frac{\left(\alpha_{l}-\alpha_{0}\right)\left(\alpha_{k}-\alpha_{0}\right)}{d \gamma}},
$$

with $f(\alpha)=d-\left(\alpha-\alpha_{0}\right)^{2} / 2 d \gamma$. Imposing that Eq. (8) is consistent with Eqs. (6), we determine the coefficients as $a_{k l}=\left(1+\sqrt{1+4 b_{k l}^{2}}\right) / 2$ and $b_{k l}=g_{k l} /\left(g_{k l}^{2}-1\right)$, where

$$
g_{k l}=\frac{\ln \left(\left|\omega_{l k}\right| / E_{c}\right)}{d \ln L} \times \begin{cases}1, & \left|\omega_{l k}\right|<E_{c}, \\ 2 / \gamma, & \left|\omega_{l k}\right|>E_{c} .\end{cases}
$$

The conditional probability to have $\alpha_{l}$ when $\alpha_{n}=\alpha$ is then

$$
P_{\alpha_{n}=\alpha}\left(\alpha_{l}\right)=\frac{P\left(\alpha, \alpha_{l}\right)}{P(\alpha)}=L^{-\frac{\left[\alpha_{l}-\alpha_{0}+g_{l n}\left(\alpha-\alpha_{0}\right)\right]^{2}}{2 d \gamma\left(1-g_{l n}^{2}\right)}} .
$$

Averaging Eq. (4) with the conditional probability Eq. (10), we find that, at a site where $\alpha_{n}=\alpha$ with $E_{n} \approx E_{F}$, the critical exchange coupling $J_{c}$ is determined by the equation

$$
\frac{1}{J_{c}}=\sum_{E_{l}=E_{F}-E_{c}}^{E_{F}+E_{c}} \frac{\left(\left|E_{l}-E_{F}\right| / E_{c}\right)^{\frac{\alpha-\alpha_{0}}{d}} L^{-\frac{d \gamma}{2}} g_{l n}^{2}}{N\left|E_{l}-E_{F}\right|} .
$$

The factor $\left(\left|E_{l}-E_{F}\right| / E_{c}\right)^{\frac{\alpha-\alpha_{0}}{d}}$ corresponds to a local pseudogap with exponent $\left(\alpha-\alpha_{0}\right) / d$ at positions where $\alpha>\alpha_{0}$. At such sites the critical exchange coupling is proportional to that exponent, in the continuum limit $\Delta \sum_{l} \rightarrow \int d E_{l}$,

$$
\frac{J_{c}(\alpha)}{D}=\frac{\alpha-\alpha_{0}}{d}\left\{1-\exp \left[-\frac{\left(\alpha-\alpha_{0}\right) \sqrt{\ln L}}{\sqrt{2 d \gamma}}\right]\right\}^{-1}
$$

Notice that when $\alpha$ takes its typical value $\alpha_{0}$, we get $J_{c}\left(\alpha_{0}\right)=$ $\sqrt{2 d \gamma / d \ln L}$, which decreases slowly with increasing $L$. At sites where $\alpha \leq \alpha_{0}$, the LDOS is larger than its typical value and $J_{c}$ vanishes. The exchange coupling $J_{c}^{(1)}$ at which there is on average only one FMM in the whole system is $J_{c}^{(1)}=J_{c}\left(\alpha_{+}\right)=\sqrt{2 \gamma} D$, where $\alpha_{+}$is obtained from the condition $P\left(\alpha_{+}\right)=1 / L^{d}$, yielding $\alpha_{+}=\alpha_{0}+\sqrt{2 \gamma} d$. Similarly, at $J_{c}^{(2)}=\sqrt{\gamma} D$, one finds $O(\sqrt{N})$ sites with FMMs. A finite density of FMMs at the AMIT is only found at very small exchange couplings, $J<J_{c}\left(\alpha_{0}\right)$, and these values are vanishingly small for $L \rightarrow \infty$.

Pseudogaps and FMMs in the insulating phase. -While we conclude that there is no finite density of FMMs at the AMIT in the infinite-volume limit, the situation changes on the insulating side of the transition. First, a hard spectral gap of order $\Delta_{I}$ quenches all magnetic moments below an exchange coupling $J_{c}^{A}$, given by Eq. (5). However, there are still power-law spectral correlations between wave functions located within the same localization volume $\xi^{d}$. One can take these correlations into account by noting that the wave function intensities within a localization volume still have a log-normal distribution with $\alpha \rightarrow \alpha_{\xi}=-\ln |\psi|^{2} / \ln \xi$. For the evaluation of $J_{c}$ we therefore should substitute $L$ by the localization length $\xi(W) \sim\left(W-W_{c}\right)^{-\nu}$. Thus, for fixed $J$, the density of FMMs depends on the localisation length $\xi$ as

$$
P_{\mathrm{FM}}=n_{\mathrm{FM}} / n_{M}=\operatorname{Erfc}\left(\sqrt{\frac{\ln \xi}{2 \gamma}} \frac{J}{D}\right) .
$$

Close to the transition, $\xi$ is large and Eq. (13) simplifies to $P_{\mathrm{FM}} \sim\left(W-W_{c}\right)^{\kappa(J)}$, decaying with an exponent $\kappa(J)=$ $(\nu d / 2 \gamma)(J / D)^{2}$.

Metallic phase. - On the metallic side of the AMIT, the correlation length $\xi_{c}$ limits the range of multifractal correlations. One can imagine the extended states close to the transition as patches of multifractal states connected to each other by tunneling [23]. This yields the scaling $|\psi|^{2 q} \sim$ $\left(\xi_{c} / L\right)^{q d} \xi_{c}^{-d-d_{q}} \sim L^{-q d}$ for $L>\xi_{c}$, leaving no finite density of FMMs in the metallic phase $\left(W<W_{c}\right)$.

Symmetry Class of the AMIT. - The magnetic scattering by FMMs changes the symmetry class of the conduction electrons from orthogonal to unitary when $\xi^{2} / \mathcal{D} \tau_{s}>1$, where $\mathcal{D}$ is the diffusion constant, $1 / \tau_{s}$ is the magnetic scattering rate, and $\xi$ denotes the correlation length on the metallic side, or the localization length on the insulating side of the transition, respectively [10, 11, 24]. The magnetic scattering rate at zero temperature is bounded from below by $2 \pi n_{F M} S(S+1)(J / D)^{2} \rho\left(\epsilon_{F}, \mathbf{r}\right) / \rho^{2}$. Coming from the metallic side of the transition, $n_{F M}$ vanishes due to the Kondo screening and the AMIT occurs at the orthogonal critical value for time-reversal symmetric systems, $W_{\mathrm{c}}^{\mathrm{O}}$. On the insulating side of the AMIT, the density of FMMs is finite, as given by Eq. (13). The LDOS at the position of the FMMs scales as $\rho\left(\epsilon_{F}, \mathbf{r}\right) \sim \xi^{d-\alpha(J)}$, where, $\alpha(J)=\alpha_{0}+d J / D$. Therefore, $1 / \tau_{s}$ is finite and, following Refs. [11, 24], we can get the critical disorder amplitude $W_{c}(J)$ from the condition $D \tau_{s}(J)=\xi\left(W_{c}(J)-W_{\mathrm{c}}^{\mathrm{O}}\right)^{2}$ :

$$
\frac{W_{\mathrm{c}}(J)-W_{\mathrm{c}}^{\mathrm{O}}}{W_{\mathrm{c}}^{\mathrm{O}}}=\left[\frac{2^{d}\left(\pi W_{c}(J) J\right)^{2} n_{M} S(S+1)}{d D^{4}}\right]^{\eta(J)},
$$

where $1 / \eta(J)=2 \nu-(d \nu / 2 \gamma)(\gamma+J / D)^{2}$. For $J$ exceeding $J^{*}=(2 \sqrt{\gamma / d}-\gamma) D$, the critical disorder amplitude $W_{\mathrm{c}}(J)$ approaches its orthogonal value $W_{\mathrm{c}}^{\mathrm{O}}$. However, for smaller exchange couplings, $J<J^{*}$, a paradoxical situation appears:

The Critical Metal Phase. - The position of the critical point $W_{\mathrm{c}}$ depends on the direction from which the AMIT is approached: Coming from the metallic side, the Kondo effect screens all magnetic moments at low temperature $T \ll T_{K}$, so that TRS is not broken and the AMIT occurs at $W_{\mathrm{c}}^{\mathrm{O}}$. Coming from the insulating side, there is a finite density of FMMs which break TRS and lead to an AMIT at the stronger disorder amplitude $W_{\mathrm{c}}(J)$ given by Eq. (14). Consistency requires then that these two distinguished critical points are connected for intermediate disorder strengths $W_{\mathrm{c}}^{\mathrm{O}}<W<W_{\mathrm{c}}(J)$ by a critical metal region. Accordingly, the mobility edge is 
extended to a critical band whose width is a function of $J$ and $n_{M}$. The resulting zero-temperature quantum phase diagram is shown in Fig. 11 with the critical metal phase appearing below the tricritical point $J^{*}$. The analytical expression for the density of FMMs compares quantitatively well with results obtained from numerical simulations of critical random banded matrices [25], and are in qualitative agreement with numerical simulations of the three-dimensional AMIT [20]. Furthermore, we have derived [25] the distribution of the Kondo temperature analytically taking into account multifractality and critical correlations of the critical wave functions and found a wide bimodal distribution at the AMIT. In two dimensions, the distribution is also bimodal with a finite width which increases with the second power of the disorder amplitude, in qualitative agreement with numerical simulations [20, 21].

A wide distribution of Kondo temperatures was argued previously to cause a finite electron dephasing rate at low temperatures [19] and to lead to deviations from the Fermi-liquid behavior [7, 9]. Here we consider the zero-temperature limit and find that on the metallic side of the AMIT the density of FMMs vanishes due to the Kondo screening. However, a finite density of FMMs survives on the insulating side of the AMIT. A new critical metal phase arises due to the interplay between the Kondo effect and the multifractality of the wave functions. One experimental consequence is the divergence of the dielectric susceptibility, $\chi(T=0) \sim \xi^{2} \sim\left(W_{c}(J)-W\right)^{-2 \nu}$ at the disorder amplitude $W_{c}(J)$ (or at a critical electron density $n_{c}(J)$ ), while the zero temperature limit of the resistivity diverges with the correlation length as $\rho(T=0) \sim \xi_{c} \sim$ $\left(W-W_{c}^{O}\right)^{-\nu}$ at the weaker disorder amplitude $W_{c}^{O}$ (or at a larger critical density $n_{c}^{O}$, accordingly). This difference $W_{c}(J)-W_{c}^{O}$ increases with the concentration of Kondo impurities as Eq. [14, maximally by about $10 \%$ [12]. We expect the critical metallic phase to be observable in materials where both the AMIT and the Kondo effect are present simultaneously at experimentally accessible temperatures, such as in amorphous metal-semiconductor alloys [3, 26] with dilute magnetic impurities 27], or in doped semiconductors, such as Si:P where thermopower measurments are consistent with the presence of Kondo impurities with $\left\langle T_{K}\right\rangle \approx 1 K[28]$.

This research was supported by the German Research Council under SFB 668 B2, the Alexander von Humboldt Foundation, the Hungarian Research Fund (OTKA) under K73361 and K75529, the International Center for Transdisciplinary Studies at Jacobs University, and the WCU program of the Korea Science and Engineering Foundation under Project No. R31-2008-000-10059-0.

[1] P. W. Anderson, Phys. Rev. 109, 1492 (1958).

[2] A. Brezini and N. Zekri, Phys. Stat. Sol. B 169, 253 (1992).

[3] B. Kramer and A. MacKinnon, Rep. Prog. Phys. 56, 1469 (1993).
[4] D. Belitz and T. R. Kirkpatrick, Rev. Mod. Phys. 66, 261 (1994).

[5] F. Evers and A. D. Mirlin, Rev. Mod. Phys. 80, 1355 (2008).

[6] P. W. Anderson, Phys. Rev. 124, 41(1961).

[7] M. Milovanovic, S. Sachdev, and R. N. Bhatt, Phys. Rev. Lett. 63, 82 (1989);

[8] M. A. Paalanen and G. A. Thomas, Helv. Phys. Acta 56, 27 (1983); M. A. Paalanen, J. E. Graebner, R. N. Bhatt, and S. Sachdev, Phys. Rev. Lett. 61, 597 (1988); S. Sachdev, Phys. Rev. B 39, 5297 (1989).

[9] E. Miranda and V. Dobrosavljević, Rep. Prog. Phys. 68, 2337 (2005).

[10] S. Hikami, A. I. Larkin, and Y. Nagaoka, Prog. Theor. Phys. 63, 707 (1980).

[11] D. Khmelnitskii and A. I. Larkin, Solid State Comm. 39, 1069 (1981).

[12] T. Ohtsuki and T. Kawarabayashi, J. Phys. Soc. Jpn. 66, 314 (1997); T. Ohtsuki, K. Slevin, and T. Kawarabayashi, Ann. Physik 8, 655 (1999).

[13] S. Kettemann and M. E. Raikh, Phys. Rev. Lett. 90, 146601 (2003).

[14] F. Wegner, Z. Phys. B 36, 209 (1980); H. Aoki, J. Phys. C 16, L205 (1983); C. Castellani and L. Peliti, J. Phys. A: Math. Gen. 19, L991 (1986); M. Schreiber and H. Grußbach, Phys. Rev. Lett. 67, 607 (1991); M. Janssen, Int. J. Mod. Phys. B 8, 943 (1994).

[15] J. T. Chalker, Physica 167A, 253 (1990); V. E. Kravtsov and K. A. Muttalib, Phys. Rev. Lett. 79, 1913 (1997); J. T. Chalker, V. E. Kravtsov, and I. V. Lerner, JETP Lett. 64, 386 (1996); V. E. Kravtsov, Ann. Phys. (Leipzig) 8, 621 (1999).

[16] E. Cuevas and V. E. Kravtsov, Phys. Rev. B 76, 235119 (2007).

[17] D. Withoff and E. Fradkin, Phys. Rev. Lett. 64, 1835 (1990); K. Ingersent, Phys. Rev. B 54, 11936 (1996); L. Fritz, S. Florens, and M. Vojta, Phys. Rev. B 74, 144410 (2006).

[18] A. C. Hewson, The Kondo Problem to Heavy Fermions, Cambridge Univ. Press (1997).

[19] S. Kettemann and E. R. Mucciolo, JETP Lett. 83, 240 (2006) [Pis'ma v ZhETF 83, 284 (2006)]; S. Kettemann and E. R. Mucciolo, Phys. Rev. B 75, 184407 (2007); T. Micklitz, T. A. Costi, and A. Rosch, ibid. B 75, 054406 (2007), T. Capron et al., ibid. 77, 033102 (2008).

[20] P. S. Cornaglia, D. R. Grempel, and C. A. Balseiro, Phys. Rev. Lett. 96, 117209 (2006).

[21] A. Zhuravlev, I. Zharekeshev, E. Gorelov, A. I. Lichtenstein, E. R. Mucciolo and S. Kettemann, Phys. Rev. Lett. 99, 247202 (2007).

[22] Y. Nagaoka, Phys. Rev. 138, 1112 (1965); H. Suhl, Phys. Rev. A 138, 515 (1965).

[23] K. B. Efetov, Supersymmetry of Disorder and Chaos (Cambridge University Press, Cambridge, 1997).

[24] T. Dröse, M. Batsch, I. Kh. Zharekeshev, and B. Kramer, Phys. Rev. B 57, 37 (1998).

[25] I. Varga, E. R. Mucciolo, and S. Kettemann, unpublished (2009).

[26] C. Van Haesendonck and Y. Bruynseraede, Phys. Rev. B 33, 1684 (1986); B. W. Dodson, W. L. McMillan, J. M. Mochel, and R. C. Dynes, Phys. Rev. Lett. 46, 46 (1981).

[27] C. C: Tsuei and R. Hasegawa, Solid State Commun. 7, 1581 (1969).

[28] M. Lakner and H. v. Lohneysen, Phys. Rev. Lett. 70, 3475 (1993). 